\title{
Balón de reanimación endovascular de aorta para pacientes en riesgo o en choque hemorrágico: experiencia en un centro de trauma de Latinoamérica
}

\author{
Resuscitative Endovascular Balloon occlusion of the Aorta in patients in \\ danger of hemorrhagic shock: experience in a Latin American trauma center \\ Juan José Meléndez¹, Carlos Alberto Ordóñez², Michael W. Parra³, Claudia Patricia Orlas, \\ Ramiro Manzano-Núñez ${ }^{5}$, Alberto Federico García ${ }^{6}$, Camilo José Salazar ${ }^{8}$, \\ María Alejandra Londoño ${ }^{8}$, Juan Esteban Ruiz ${ }^{8}$, José Julián Serna ${ }^{1}$, Edison Angamarca ${ }^{1}$, \\ Alexander Salcedo' ${ }^{1}$ Camilo Andrés Peña ${ }^{1}$, Fernando Rodríguez ${ }^{7}$ \\ 1 Fellow de Cirugía de Trauma y Emergencias, Universidad del Valle, Cali, Colombia \\ 2 MD, FACS; jefe, Sección de Cirugía de Trauma y Emergencias, Fundación Valle del Lili; Departamento de Cirugía, Universidad del \\ Valle-Universidad ICESI, Cali, Colombia \\ 3 MD, FACS; Department of Trauma Critical Care, Broward General Level I Trauma Center, Fort Lauderdale, Florida, USA \\ 4 Médico asistente de investigación, Centro de Investigaciones Clínicas, Fundación Valle del Lili, Cali, Colombia \\ 5 Médico asistente de investigación, Centro de Investigaciones Clínicas, Fundación Valle del Lili, Cali, Colombia \\ 6 Médico, cirujano de Trauma, Emergencias y Cuidado Intensivo; profesor asociado, Universidad del Valle, Cali, Colombia; profesor \\ asociado, Universidad ICESI, Fundación Valle del Lili, Cali, Colombia \\ 7 Cirujano de Trauma y Emergencias, Fundación Valle del Lili, Cali, Colombia \\ 8 Estudiante de Medicina, Universidad ICESI, Cali, Colombia
}

\section{Resumen}

Introducción. La maniobra de reanimación mediante el denominado "balón de reanimación endovascular de aorta"(Resuscitative Endovascular Balloon Occlusion of the Aorta, REBOA), es un procedimiento para obtener el control proximal de la hemorragia. Nuestra hipótesis es que puede usarse como una maniobra emergente para el manejo del choque hemorrágico y como una intervención para prevenir la aparición de hemorragia masiva en los pacientes en riesgo.

Materiales y métodos. Se recolectaron los datos de una cohorte prospectiva de pacientes entre el 2014 y 2018 en un centro de trauma de nivel I del suroccidente colombiano. Los datos se presentaron, de acuerdo con su distribución de normalidad, en medias y desviaciones estándar o medianas y rangos intercuartílicos.

Resultados. Se incluyeron 70 pacientes, 27 considerados en riesgo de choque hemorrágico (mujeres embarazadas con placentación anormal) y 43 con choque hemorrágico (pacientes de trauma sometidos a cirugía y oclusión aórtica con balón). En el primer grupo, ningún paciente requirió transfusión masiva y no se reportaron muertes. En el último, hubo aumento significativo de la presión arterial sistémica después del procedimiento de 50,I $\pm 22,5$ a I07 mm Hg (rango: 87-I29) y la mortalidad fue del 30,2 \%.

Fecha de recibido: 8/06/2018 - Fecha aceptación: 28/08/2018

Correspondencia: Carlos Alberto Ordóñez, Carrera 98 № 18-49, Fundación Valle del Lili, Cali, Colombia, Teléfono: (300) $631-9118$

Correo electrónico: ordonezcarlosa@gmail.com

Citar como: Meléndez JJ, Ordóñez CA, Parra MW, Orlas CP, Manzano-Núñez R, García AF, et al. Balón de reanimación endovascular de aorta para pacientes en riesgo de o en choque hemorrágico: experiencia en un centro de trauma de Latinoamérica. Rev Colomb Cir. 2019;34:124-31. https://doi.org/10.30944/20117582.106

Este es un artículo de acceso abierto bajo una Licencia Creative Commons - BY-NC-ND https://creativecommons.org/licenses/by-nc-nd/4.0/deed.es 
Conclusión. Esta muestra de pacientes sometidos a reanimación mediante oclusión aórtica con REBOA es la más grande reportada hasta ahora en Latinoamérica. Esta es una herramienta eficaz para el manejo de pacientes en choque hemorrágico o en riesgo de uno.

Palabras clave: aorta; procedimientos endovasculares; oclusión con balón; heridas y lesiones; hemorragia; transfusión sanguínea; reanimación cardiopulmonar.

\begin{abstract}
Introduction: Resuscitative Endovascular Balloon Occlusion of the Aorta (REBOA) is a procedure that involves placement of an endovascular balloon in the aorta to obtain proximal control of hemorrhage. We hypothesize that the REBOA can be used as an emergent maneuver for the management of patients with hemorrhagic shock and as a prophylactic intervention to prevent the appearance of massive hemorrhage in populations at risk.

Methods: Data were collected from a prospective cohort of patients in the period between 2014 and 20I8, at a level I trauma center in Southwestern Colombia. The data was presented according to their distribution of normality, in means and standard deviations or medians and interquartile ranges.

Results: Seventy patients were included, 27 considered at risk of hemorrhagic shock (pregnant women with abnormal placentation) and 43 with hemorrhagic shock (trauma patients undergoing surgery and REBOA). In the first group, no patient required massive transfusion and no deaths were reported. In the latter, there was a significant increase in blood pressure [SBP pre-REBOA: $50.1 \pm 22.5$, post-REBOA SBP: IO7 (87-I29)] and mortality was 30.2\%. Conclusion: Our experience in the use of REBOA is the largest reported in Latin America. This is an effective tool for the management of patients at or at risk of hemorrhagic shock.
\end{abstract}

Key words: aorta; endovascular procedures; balloon occlusion; wounds and injuries; hemorrhage; blood transfusion; cardiopulmonary resuscitation.

\section{Introducción}

Las muertes por hemorragia representan un problema global, con un estimado de I,9 millones de muertes por año, de las cuales I, 5 millones son secundarias al trauma. Debido a que este problema afecta de manera desproporcionada a personas jóvenes, estos 1,5 millones de muertes equivalen a 75 millones de años de vida perdidos ${ }^{\mathrm{I}}$. El control de la hemorragia, la corrección de la coagulopatía, el mantenimiento de la perfusión tisular mediante reanimación con fluidos y el manejo de la normotermia, constituyen las principales herramientas para el manejo de pacientes graves con choque hemorrágico ${ }^{2}$.

El manejo actual de la hemorragia no compresible del torso (Non Compressible Torso Hemorrhage, NCTH) es bastante invasivo. Para controlar la hemorragia intraabdominal, se practica una laparotomía, mientras que los pacientes que presentan o progresan a choque hemorrágico persistente, son candidatos a toracotomía abierta para pinzar la aorta y lograr la reanimación cardiovascular ${ }^{3}$.

La oclusión aórtica con balón intravascular, una maniobra de reanimación mediante el denominado "balón de reanimación endovascular de aorta" (Resuscitative Endovascular Balloon Occlusion of the Aorta, REBOA), es un procedimiento que consiste en colocar e inflar un balón dentro de la aorta para obtener el control proximal de la hemorragia ${ }^{45}$. Es menos invasivo que la reanimación mediante toracotomía, y se ha venido implementando para pinzar la aorta y reanimar pacientes en paro cardiaco de origen traumático ${ }^{6}$. Además, ha surgido 'información' que sustenta su uso como una herramienta eficaz para casos diferentes al trauma, por ejemplo, el control de la hemorragia en mujeres embarazadas que son sometidas a cesárea por placentación anormal y para el manejo de la ruptura de aneurismas de aorta abdominal 2,78 . 
El control proactivo del sangrado y la reanimación del sistema cardiovascular que provee este procedimiento, podrían ser de importancia incalculable para mitigar los efectos deletéreos de la hemorragia no controlada y, más aún, para prevenir su aparición.

Nuestra hipótesis es que la reanimación con REBOA puede usarse de forma efectiva y segura como una maniobra emergente en los pacientes con choque hemorrágico y como una intervención para prevenir la aparición de hemorragia masiva en las poblaciones en riesgo.

\section{Materiales y métodos}

Previa aprobación del protocolo de investigación por el comité de revisión institucional, se hizo un estudio prospectivo de cohorte de enero del 2014 a marzo del 2018 en un centro de trauma de nivel I ubicado en el suroccidente colombiano, en el que se recolectaron los datos de los pacientes que cumplían con los criterios de selección.

Los pacientes elegibles fueron aquellos que a su ingreso a la institución estaban en riesgo de presentar o habían presentado choque hemorrágico. Los pacientes con choque hemorrágico fueron las víctimas de trauma que al ingreso a la institución se les diagnosticó sangrado activo a través de una cavidad y presentaron como consecuencia choque hemorrágico grado III-IV, mientras que las pacientes en riesgo de choque hemorrágico fueron mujeres embarazadas con implantación anómala de la placenta (placenta accreta, increta o percreta). Estas fueron diagnosticadas previamente mediante resonancia magnética y, según el protocolo institucional de acretismo placentario, fueron programadas para cesárea electiva con colocación simultánea del REBOA.

La colocación del REBOA se utilizó de forma emergente y como medida profiláctica. Por lo tanto, su uso en mujeres embarazadas con parto por cesárea programada, fue una medida preventiva de sangrado masivo, mientras que, en los pacientes de trauma, fue usado como método para brindar soporte hemodinámico y, simultáneamente, para el control proximal de la hemorragia.

\section{Análisis estadístico}

Los datos fueron analizados utilizando el programa Stata $\mathrm{I}_{4} \mathrm{O}^{\mathrm{TM}}$. Las variables categóricas (cualitativas) se presentan en frecuencias absolutas y relativas; las variables continuas (cuantitativas) se presentan con medidas de tendencia central y de dispersión.

Los valores que cumplían con los supuestos de normalidad, se presentan con medias y desviación estándar, y aquellos que no lo hacían se resumen en medianas y rango intercuartílico.

El análisis de los dos subgrupos se hizo de forma independiente $\mathrm{y}$, para aquel cuyo $\mathrm{n}$ era mayor de 30, se aplicó el test de normalidad de Shapiro Wilk, considerando que se trataba de una distribución normal aquellas variables cuya $\mathrm{p}$ fuera mayor de 0,05 .

\section{Resultados}

Se recolectaron los datos de 70 pacientes, 27 considerados en riesgo de choque hemorrágico (mujeres embarazadas con placentación anormal) y 43 con choque hemorrágico (pacientes de trauma sometidos a cirugía y oclusión con balón de la aorta).

En el primer grupo $(n=27$ mujeres embarazadas), la media de edad fue de 34,I \pm 5,5 años, la mediana de la edad de gestación fue de 35 semanas (rango, 32 a 36), el tipo de alteración placentaria que se diagnosticó con mayor frecuencia fue la placenta accreta $(\mathrm{n}=2 \mathrm{I}, 78 \%)$, percreta $(n=I$, $4 \%)$, previa ( $\mathrm{n}=5, \mathrm{I} 8 \%)$, la mediana del $\mathrm{pH}$ intraoperatorio fue de 7,36 (rango, 7,34 a 7,38 ) y la mediana de la hemorragia intraoperatoria fue de I.800 $\mathrm{ml}$ (rango, I.200 a 2.500). Ninguna de las pacientes requirió transfusión masiva, solo se transfundieron dos unidades de glóbulos rojos en las primeras 24 horas, y se observó disminución de un gramo de hemoglobina a las 24 horas del posoperatorio con respecto a la medida inicial (al ingreso: $\mathrm{IO}, 7 \pm \mathrm{I}, 8 \mathrm{~g} / \mathrm{dl}$; a las 24 horas: $9,8 \pm$ I,8 g/dl) (tabla I).

En el segundo grupo $(\mathrm{n}=43$ pacientes con choque hemorrágico), la mediana de edad fue de 32 años (rango, 23 a 45), la mayoría fueron hombres $(\mathrm{n}=32,79 \%)$. El mecanismo de trauma más frecuente fue el penetrante, $29(67,4 \%)$, y hubo 7 
Tabla 1. Características y variables clínicas de mujeres con placentación anormal y colocación del REBOA $(\mathrm{N}=27)$

\begin{tabular}{ll}
\hline Edad (años) (DE) & $34,1(5,5)$ \\
Edad gestacional (semanas) (RIQ) & $35(32-36)$ \\
Placenta accreta [n (\%)] & $21(78 \%)$ \\
Placenta percreta [n (\%)] & $1(4 \%)$ \\
Placenta previa [n (\%)] & $5(18 \%)$ \\
pH intraoperatorio mediana (RIQ) & $7,36(7,34-7,38)$ \\
$\begin{array}{l}\text { Hemorragia intraoperatoria (ml), } \\
\text { mediana (RIQ) }\end{array}$ & $1.800(1.200-2.500)$ \\
$\begin{array}{l}\text { Unidades de glóbulos rojos en } 24 \\
\text { horas, mediana (RQI) }\end{array}$ & $2(0-3)$ \\
$\begin{array}{l}\text { Unidades de plasma en 24 horas, } \\
\text { mediana }\end{array}$ & 3 \\
$\begin{array}{l}\text { Unidades de plaquetas en 24 horas, } \\
\text { mediana }\end{array}$ & 4 \\
$\begin{array}{l}\text { Unidades de crioprecipitados en } 24 \\
\text { horas, mediana } \\
\text { Cristaloides en } 24 \text { horas (ml), } \\
\text { mediana (RIQ) }\end{array}$ & 5 \\
$\begin{array}{l}\text { Hemoglobina de ingreso (g/dl), } \\
\text { mediana (DE) } \\
\text { Hemoglobina a las 24 horas (g/dl), } \\
\text { mediana (DE) }\end{array}$ & $9.3(1,8)$ \\
\hline
\end{tabular}

DE: desviación estándar; RIQ: rango intercuartílico

heridas por arma corto-punzante y 22 por arma de fuego. La mayoría de los pacientes sufrieron trauma grave, con un ISS (Injury Severity Score) de 25 (rango, 25-4I). La mediana de unidades de glóbulos rojos transfundidas en las primeras 24 horas fue de 6 (rango, 4-I3) y la de cristaloides perfundidos en las primeras 24 horas fue de $4.715 \mathrm{ml}$ (rango, 3.225-6.200). A su ingreso al quirófano, todos los pacientes tenían una presión arterial sistólica por debajo de $90 \mathrm{~mm} \mathrm{Hg}(55,7 \pm 27,4)$ (tabla 2).

En relación con las variables asociadas con la oclusión aórtica con REBOA (tabla 3), el método de acceso arterial usado con mayor frecuencia en las mujeres embarazadas fue el percutáneo $(n=25$; 92,5\%), mientras que, en las víctimas de trauma, fue la vía abierta mediante disección quirúrgica $(\mathrm{n}=37 ; 86,0 \%)$. En todas las mujeres embarazadas, el balón se colocó en la zona III; en las víctimas de trauma, los balones se colocaron así: 2I (48,8 \%) en la zona I, 5 (II,6 \%) en la zona III, y I7 (39,5\%) en las zonas I y III.
Tabla 2. Características de los pacientes de trauma manejados con balón intraaórtico $(\mathrm{N}=43)$

\begin{tabular}{|c|c|}
\hline Edad (años), mediana (RIQ) & $32(23-45)$ \\
\hline Sexo masculino [n (\%)] & $34(79)$ \\
\hline Trauma cerrado [n (\%)] & $14(32,5)$ \\
\hline Trauma penetrante $[\mathrm{n}(\%)]$ & $29(67,4)$ \\
\hline $\operatorname{HPAC}^{*}(\mathrm{n})$ & 7 \\
\hline $\operatorname{HPAF}^{*}(n)$ & 22 \\
\hline Injury Severity Score (RIQ) & $25(25-41)$ \\
\hline $\begin{array}{l}\text { Unidades de glóbulos rojos en } \\
24 \text { horas, mediana (RQI) }\end{array}$ & $6(4-13)$ \\
\hline $\begin{array}{l}\text { Unidades de plasma en } 24 \text { horas, } \\
\text { mediana (RIQ) }\end{array}$ & $7(4-12)$ \\
\hline $\begin{array}{l}\text { Unidades de plaquetas en } 24 \text { horas, } \\
\text { mediana (RIQ) }\end{array}$ & $6(0-12)$ \\
\hline $\begin{array}{l}\text { Unidades de crioprecipitados en } 24 \\
\text { horas, mediana (RIQ) }\end{array}$ & $10(4-18)$ \\
\hline $\begin{array}{l}\text { Cristaloides en } 24 \text { horas (ml), } \\
\text { mediana (RIQ) }\end{array}$ & $4.715(3.225-6.200)$ \\
\hline $\begin{array}{l}\text { Presión arterial sistólica al ingreso al } \\
\text { quirófano, mediana (DE) }\end{array}$ & $55,7(27,4)$ \\
\hline
\end{tabular}

RIQ: rango intercuartílico; DE: desviación estándar; HPAC: herida por arma cortopunzante; HPAF: herida por proyectil de arma de fuego.

La mayoría de las pacientes en riesgo de choque hemorrágico sometidas al procedimiento presentaba estabilidad hemodinámica, con mediana de presión arterial sistólica de IO8 (rango, 87-II9) mm Hg. Por el contrario, la mayoría de las víctimas de trauma tenía una media de presión arterial sistólica por debajo de $60 \mathrm{~mm} \mathrm{Hg}(50, \mathrm{I} \pm 22,5)$, inmediatamente antes de colocar el balón intraaórtico, la cual aumentó alrededor del $50 \%$ después de inflarlo, con una mediana de I07 (rango, 87-I29) $\mathrm{mm} \mathrm{Hg.}$

En ambos grupos, el método más usado para confirmar una adecuada posición del balón intraaórtico, fue el examen clínico: I4 (52 \%) mujeres embarazadas y 42 (98\%) pacientes de trauma. La mediana del tiempo de oclusión fue considerablemente mayor en estos últimos: 4I minutos (rango, 25-60) en víctimas de trauma y 25 minutos (rango, 20-30) en mujeres embarazadas.

En el grupo de los pacientes de trauma hubo con frecuencia lesiones asociadas de alto grado: 
Tabla 3. Variables relacionadas con la colocación del balón intraaórtico

\begin{tabular}{|c|c|c|}
\hline & $\begin{array}{c}\text { Mujeres } \\
\text { embarazadas } \\
\text { con } \\
\text { placentación } \\
\text { anormal } \\
(\mathrm{N}=27) \\
\mathrm{n}(\%)\end{array}$ & $\begin{array}{c}\text { Pacientes } \\
\text { de trauma } \\
(\mathrm{N}=43) \\
\mathrm{n}(\%)\end{array}$ \\
\hline \multicolumn{3}{|l|}{ Método para acceso arterial } \\
\hline Percutáneo & $25(92,5)$ & $6(14)$ \\
\hline Disección quirúrgica & $2(7,4)$ & $37(86,0)$ \\
\hline \multicolumn{3}{|l|}{ Zona de colocación } \\
\hline I & & $21(48,8)$ \\
\hline III & $27(100)$ & $5(11,6)$ \\
\hline I y III & & $17(39,5)$ \\
\hline \multicolumn{3}{|l|}{ Presión arterial sistólica } \\
\hline $\begin{array}{l}\text { Previa }(\mathrm{mm} \mathrm{Hg}) \text {, mediana } \\
\text { (RIQ) }\end{array}$ & $108(87-119)$ & $50,1(22,5)$ \\
\hline $\begin{array}{l}\text { Posterior (mm Hg), mediana } \\
\text { (RIQ) }\end{array}$ & $118(110-126)$ & $107(87-129)$ \\
\hline \multicolumn{3}{|l|}{ Confirmación de la colocación } \\
\hline Por clínica & $14(52)$ & $42(98)$ \\
\hline Por fluoroscopia & $13(48)$ & $1(2)$ \\
\hline $\begin{array}{l}\text { Tiempo de oclusión de } \\
\text { la aorta (minutos), mediana } \\
\text { (RIQ) }\end{array}$ & $25(20-30)$ & $41(25-60)$ \\
\hline
\end{tabular}

pulmonar en 5 pacientes, esplénica en 2, hepática en 8 y renal en I. Se presentaron heridas en algún vaso axial del torso en 28 (65,I \%) de los pacientes: aorta torácica en 3, arteria pulmonar en 3 , arteria subclavia en 5, arteria innominada en I, arteria mamaria en 4, arteria mesentérica en 3, arteria iliaca en 8 , arteria axilar en I; además, un paciente presentó lesión de la vena cava retrohepática. Nueve pacientes ingresaron al servicio con pelvis inestable (tabla 4).

En el grupo de mujeres embarazadas, ninguna presentó falla orgánica en el día 2 ni en el día 5: SOFA (Sequential Organ Failure Assessment) de o (rango, o-2). Solo cuatro requirieron asistencia respiratoria mecánica. La mediana de estancia hospitalaria fue de 5 (rango, 3-Io) días. No hubo complicaciones asociadas con el acceso vascular del REBOA ni se requirió soporte con diálisis; además, no se reportó ninguna muerte.
Tabla 4. Descripción de las lesiones de alto grado (escala de gravedad en trauma American Association for the Surgery of Trauma) de víscera sólida y de lesiones vasculares, en los pacientes con trauma sometidos a oclusión aórtica con balón intravascular $(\mathrm{N}=43)$

\begin{tabular}{lc}
\hline Lesión & 5 \\
\hline Pulmonar de grado IV/V & 2 \\
Esplénica de grado IV/V & 8 \\
Hepática de grado IV/V & 1 \\
Renal de grado IV & 28 \\
Herida en vaso axial del torso & 3 \\
Aorta torácica & 3 \\
Arteria pulmonar & 5 \\
Arteria subclavia & 1 \\
Arteria innominada & 4 \\
Arteria mamaria & 3 \\
Arteria mesentérica & 8 \\
Arteria iliaca (común, externa o interna) & 1 \\
Arteria axilar & 1 \\
Vena cava retrohepática & 9 \\
Pelvis inestable &
\end{tabular}

En el grupo de pacientes de trauma en choque hemorrágico, la mayoría desarrolló falla orgánica múltiple en el segundo día del posoperatorio [SOFA: 6 (rango, 2-6)]; sin embargo, el puntaje disminuyó el $50 \%$ del valor inicial al quinto día [SOFA: 3 (rango, 2-6)]. La mediana de la estancia en la unidad de cuidados intensivos fue de 4 días (rango, 2-7) y, la de la estancia hospitalaria, de II días (rango, 5-25). Cuatro (9,3\%) pacientes presentaron complicaciones relacionadas con el acceso vascular (trombosis arterial en tres e isquemia en uno), 5 (II,6\%) requirieron diálisis y I3 (30,2 \%) fallecieron (tabla 5).

\section{Discusión}

Nuestra hipótesis es que la reanimación mediante el uso del REBOA puede ser usada como una maniobra emergente, para el manejo de los pacientes con choque hemorrágico, y como una 
Tabla 5. Resultados de los pacientes en riesgo de o en choque hemorrágico, sometidos a oclusión aórtica con balón intravascular

\begin{tabular}{lcc}
\hline & \multicolumn{2}{c}{ Mujeres } \\
& $\begin{array}{c}\text { embarazadas } \\
\text { con placentación } \\
\text { anormal } \\
\text { (N=27) }\end{array}$ & $\begin{array}{c}\text { Pacientes } \\
\text { (N=43) }\end{array}$ \\
\hline SOFA día 2, mediana (RIQ) & $0(0-2)$ & $6(2-6)$ \\
SOFA día 5, mediana (RIQ) & $0(0-2)$ & $3(2-6)$ \\
$\begin{array}{l}\text { Estancia en UCl (días), } \\
\text { mediana (RIQ) }\end{array}$ & $1(1-2)$ & $7(4-19)$ \\
$\begin{array}{l}\text { Asistencia respiratoria, } \\
\text { mecánica (días) }\end{array}$ & $4(\%)$ & $4(2-7)$ \\
$\begin{array}{l}\text { Estancia hospitalaria (días), } \\
\text { mediana (RIQ) }\end{array}$ & $5(3-10)$ & $11(5-25)$ \\
$\begin{array}{l}\text { Complicaciones relacionadas } \\
\text { con el procedimiento [n (\%)] }\end{array}$ & $0(0)$ & $4(9,3)$ \\
Necesidad de diálisis [n (\%)] & $0(0)$ & $5(11,6)$ \\
Mortalidad [n (\%)] & $0(0)$ & $13(30,2)$ \\
\hline
\end{tabular}

SOFA: Sequential Organ Failure Assessment; UCI: unidad de cuidados intensivos; RIQ: rango intercuartílico

intervención profiláctica para prevenir la aparición de hemorragia masiva en las poblaciones en riesgo.

Los hallazgos de este estudio sugieren que la oclusión aórtica con REBOA es una estrategia segura y eficaz para el manejo proactivo de los pacientes con riesgo de hemorragia potencialmente fatal.

En el grupo de mujeres embarazadas con placentación anormal, ninguna requirió transfusión masiva de glóbulos rojos [mediana de glóbulos rojos transfundidos en las primeras 24 horas: 2 (rango, 0-3)], las cifras de tensión arterial sistólica se mantuvieron por encima de $90 \mathrm{~mm} \mathrm{Hg}$ a lo largo del procedimiento [valor previo: Io8 (rango, 87-II9) mm Hg; valor posterior: $\mathrm{IO} 7$ (rango, 87-I29) $\mathrm{mm} \mathrm{Hg}$ ], no se reportaron complicaciones relacionadas y no se presentaron casos de mortalidad.

Para el grupo de pacientes con trauma grave y en choque hemorrágico, los resultados muestran que es una estrategia adjunta efectiva en la reanimación y el control mecánico de la hemorragia, que produce un aumento de más del $50 \%$ en las cifras de presión arterial sistólica después de su colocación [valor previo: $50, \mathrm{I} \pm 22,5 \mathrm{~mm} \mathrm{Hg}$; valor posterior: $\mathrm{IO} 7 \mathrm{~mm} \mathrm{Hg}$ (rango, 87-I29)], una reducción llamativa en el puntaje de la valoración de falla orgánica, SOFA de 6,I $\pm 2,6$ en el día 2 y de 3 en el día 5 (rango, 2-6) y una proporción de mortalidad aceptable $(\mathrm{n}=\mathrm{I} 3 ; 30,2 \%)$ considerando la gravedad de las lesiones que presentan estos pacientes.

Hay vasta información que sugiere que la reanimación mediante oclusión con el REBOA debe ser considerada una estrategia adjunta de la reanimación de control de daños, en trauma, especialmente, cuando es difícil encontrar el origen real de la hemorragia y se requieren procedimientos adicionales, como la formación de émbolos (embolization) o el traslado a una sala de radiología intervencionista, a pesar de la inestabilidad hemodinámica que pueda presentar el paciente. Para casos como estos, la oclusión aórtica con balón intravascular podría considerarse una buena estrategia adjunta que ayuda a resolver la situación ${ }^{2}$.

En otras circunstancias clínicas, como trauma o sangrado de origen médico asociado con paro cardiaco, hay resultados prometedores, que reportan supervivencia en el $57 \%$ de los pacientes, relativamente más alta al compararla con otras estrategias convencionales como la toracotomía de resucitación ${ }^{6}$. Hay otras ocasiones en que su uso es bastante controversial, especialmente, cuando las heridas se ubican por encima del diafragma. En una publicación reciente de una serie de casos de pacientes con trauma torácico, se describe el uso del REBOA combinado con la esternotomía media como una estrategia factible para el abordaje de estas lesiones, con la cual, 6 de 7 pacientes sobrevivieron a los 30 días de seguimiento 9.

Para el manejo profiláctico de la hemorragia posparto en mujeres con alteraciones de la implantación placentaria, la evidencia es creciente. En un metaanálisis reciente, se reporta que el REBOA como estrategia adjunta para prevenir la hemorragia se ha asociado con un menor volumen de hemorragia intraoperatoria y una menor necesidad de transfusiones sanguíneas ${ }^{7,8}$. 
En cuanto a la incidencia de complicaciones, hay evidencia fuerte que sugiere que estas son escasas y, en otro metaanálisis que incluyó 13 estudios con 424 pacientes, hubo una incidencia global de complicaciones entre 4 y $5 \%$, sin heterogeneidad significativa ${ }^{\text {Io }}$. Esta descripción sustenta la mayoría de los hallazgos reportados en este estudio.

A pesar de que el tamaño de la muestra no permite un mayor efecto de sus resultados, en este estudio se aborda un campo innovador; se expande el campo del cirujano de trauma y emergencias pues, en todos los casos, fue este profesional, con entrenamiento en cirugía endovascular, quien colocó e infló el balón en la aorta.

Esto es de vital importancia porque, tradicionalmente, este procedimiento es realizado por un equipo multidisciplinario, con un cirujano vascular y un radiólogo intervencionista, en salas híbridas o de radiología convencional, lo cual implica altos costos y es poco factible en los países de recursos limitados.

El hecho de que un cirujano sea capaz de realizar todo el procedimiento -inflar adecuadamente el balón intraaórtico, comprobar su adecuada colocación, apoyar al equipo quirúrgico si se requiere, contribuir a un buen seguimiento y vigilancia de la reanimación, y retirar el catéter reduciendo al mínimo las complicaciones- finalmente, se traduce en que la intervención tenga un futuro prometedor. Esto es especialmente cierto cuando las condiciones son austeras y, si se cuenta con un cirujano de trauma entrenado, se puede optar por alternativas como esta para manejar pacientes con trauma y choque hemorrágico, o prevenir una hemorragia que podría implicar la pérdida de dos vidas.

En estudios futuros se debe continuar abordando los tópicos que aún son controversiales, pero, especialmente, se deben establecer cuáles son las variables clínicas que podrían, en algún momento, ayudar a identificar de forma más temprana a quienes más se beneficiarían con esta intervención realmente.

Esta es la serie más grande reportada en Latinoamérica sobre la reanimación mediante el uso de REBOA. Nuestros hallazgos sugieren que el REBOA puede ser una herramienta eficaz para la reanimación y el control oportuno de la hemorragia en pacientes de trauma con choque hemorrágico, como también, de manera profiláctica, en pacientes con riesgo de sufrir esta condición.

La principal limitación del presente estudio es su naturaleza y el tamaño de la muestra, por lo cual los efectos observados de la intervención solo tendrían validez interna, es decir que se esperaría obtener resultados similares si se logran reproducir condiciones iguales a las planteadas aquí. La naturaleza observacional del estudio impide comparar esta intervención con otras convencionales; sin embargo, con la inclusión continua de pacientes será posible obtener un tamaño de muestra mayor, para establecer medidas de asociación que permitan aumentar el peso de los hallazgos descritos.

En resumen, esta es la serie más grande reportada en Latinoamérica sobre la reanimación mediante oclusión aórtica con balón endovascular. Sus hallazgos sugieren que puede ser utilizada como una herramienta eficaz para la reanimación y el control oportuno de la hemorragia en pacientes de trauma con choque hemorrágico, como también, de manera profiláctica, en pacientes con riesgo de sufrir esta condición.

Conflictos de intereses: ninguno declarado.

Fuentes de financiación: autofinanciado.

\section{Referencias}

I. Lee SW, Shin SW. Hemorrhagic shock. N Engl J Med. 2018;378:1850-3.

2. Saito N, Matsumoto H, Yagi T, Hara Y, Hayashida K, Motomura T, et al. Evaluation of the safety and feasibility of resuscitative endovascular balloon occlusion of the aorta. J Trauma Acute Care Surg. 2015;78(5):897-904.

3. Fontenelle Ribeiro Jr MA, Brenner M, Nguyen A, Feng YD, Reis de-Moura R, Rodrigues VC, et al. Oclusão ressuscitativa por meio de balão endovascular da aorta ( REBOA ): revisão atualizada . Rev Col Bras Cir. 2018;45:eI709. doi: I0.1590/oroo-6991e-20181709

4. Manzano R, Naranjo MP, Foianini E, Ferrada P, Rincón E, García HA, et al. A meta-analysis of resuscitative 
endovascular balloon occlusion of the aorta (RE$\mathrm{BOA}$ ) or open aortic cross-clamping by resuscitative thoracotomy in non-compressible torso hemorrhage patients. World J Emerg Surg. 20I7;I2:I-9. doi: I0.II86/ SI3OI7-OI7-OI42-5

5. Stannard A, Eliason JL, Rasmussen TE. Resuscitative endovascular balloon occlusion of the aorta (REBOA) as an adjunct for hemorrhagic shock. J Trauma. 2OII;71:I869-72.

6. Brenner M, Teeter W, Hoehn M, Pasley J, Hu P, Yang $\mathrm{S}$, et al. Use of fresuscitative endovascular balloon occlusion of the aorta for proximal aortic control in patients with severe hemorrhage and arrest. JAMA Surg. 2018;153:130-5.

7. Ordóñez CA, Manzano-Núñez R, Parra MW, Rasmussen TE, Nieto AJ, Herrera-Escobar JP, et al. Prophylactic use of resuscitative endovascular balloon occlusion of the aorta in women with abnormal placentation: A systematic review, meta-analysis, and case series. J Trauma Acute Care Surg. 20I8;84:809-I8.
8. Manzano-Núñez R, Escobar-Vidarte MF, Naranjo MP, Rodríguez F, Ferrada P, Casallas JD, et al. Expanding the field of acute care surgery: A systematic review of the use of resuscitative endovascular balloon occlusion of the aorta (REBOA) in cases of morbidly adherent placenta. Eur J Trauma Emerg Surg. 2018;44:519-26. doi: I0.I007/sooo68-0I7-0840-4

9. Ordóñez CA, Parra MW, Manzano-Núñez R, Herrera-Escobar JP, Serna JJ, Rodríguez-Ossa P, et al. Intraoperative combination of resuscitative endovascular balloon occlusion of the aorta and a median sternotomy in hemodynamically unstable patients with penetrating chest trauma: Is this feasible? J Trauma Acute Care Surg. 20I8;84:752-7. doi: I0.I097/TA.000000000000I807

Io. Manzano-Núñez R, Orlas CP, Herrera-Escobar JP, Galvagno S, DuBose J, Meléndez JJ, et al. A meta-analysis of the incidence of complications associated with groin access after the use of resuscitative endovascular balloon occlusion of the aorta in trauma patients. J Trauma Acute Care Surg. 2018;85:626-34. doi: Io.I097/ TA.ooooooooooool978 